\title{
An experimental investigation of collapsible tube flows at the onset of self-excited oscillations
}

\author{
V. Oruç ${ }^{1} \&$ M. Ö. Çarpınlıŏlu ${ }^{2}$ \\ ${ }^{I}$ Department of Mechanical Engineering, University of Dicle, Turkey \\ ${ }^{2}$ Department of Mechanical Engineering, University of Gaziantep, Turkey
}

\begin{abstract}
In this paper, suddenly arisen self-excited oscillations which were observed during airflow through some collapsible rubber tubes are analysed and evaluated without paying attention to the mechanisms originating the oscillations. The principal experimental results are the pressure and velocity measurements corresponding to the instant at which oscillations are emerged and frequency determined from those measurements. The time-dependent pressure values were obtained by means of pressure transducers while a hot wire anemometer was used for measuring the velocity of the flow. It was verified that the flow had been highly unsteady and chaotic during the oscillations. The variety of experimental parameters (tube material, tube-wall thickness, tube length, flow rate) presented in the study should be useful on account of acquiring more general results related to the subject. It had been noticed that all of these parameters directly affected behaviour of the oscillations and conditions of their appearance; therefore it has been emphasized that they should be carefully taken into consideration in the collapsible tube flow investigations. The results of this study should also be important in of using air instead of water which had been widely preferred as fluid almost in all of the previous experimental investigations.

Keywords: transmural pressure, fluid-solid interaction, self-excited oscillations, Starling resistor, collapsible tube flow, data acquisition, Womersley number.
\end{abstract}




\section{Introduction}

The flow in collapsible tubes has attracted attention of the researchers due to its close relevance to physiological flows. Such flows can be investigated experimentally in the laboratory. An interesting and usual case, which is related to flows through similar structures, is the emergence of audible self-excited oscillations as a result of negative transmural (internal minus external) pressure across the tube wall. An interaction takes place between tube-wall mechanics and flow dynamics in the collapsible tube flows. The self-excited oscillations arise due to this interaction and the flow has therefore a complex structure. The oscillations are especially influenced by both of fluid and tube properties and they have a wide range of frequency. Many researchers had studied the topic theoretically (for example, Cancelli and Pedley [1]) or experimentally (e.g., Conrad [2]) to find out the possible mechanisms causing the oscillations to occur. In the experimental works water was mostly used as flowing fluid (Ohba et al [3]), however in a few studies air had been preferred as fluid (Gavriely et al [4]).

The collapsible tube flow studies have a great importance in the biomechanical and biomedical applications. The physiological examples of the collapsible tube flows can be given as follows: The Korotkoff sounds heard while measuring blood pressure (Bertram et al [5]) are directly related to the self-excited oscillations originated in the veins of the arm. Griffiths [6] emphasized that urethra behaves like a collapsible tube. Furthermore flow in the lung is seriously influenced by the collapsibility of the lung airways. It has been thought by Gavriely et al [7] that the self-excited oscillations occurred in the collapsible lung airways have different respiration sounds. Gavriely and Jensen [8] stated that the origin of snoring sounds during sleep is depended on the flow-induced deformation of soft palate and pharynx wall.

In the presented study airflow through various collapsible tubes which are under the effect of external pressure is aimed to be investigated experimentally. The main findings are offered as the values corresponding just to the instant of origination of oscillations. The experimental parameters related to tube properties and flow rate have been selected in a wide range; the results are compared and discussed.

\section{Experimental set-up}

The open-system experimental set-up in which air was used as fluid is introduced in the paper of Oruç and Çarpınlıoğlu [9] in detail. Therefore only brief information about the system is given in this study. The collapsible tube, which was horizontally placed in an airtight box, has been attached to rigid pipe segments with clamps at its both ends. Airflow was supplied steadily to the system by means of a screw-type compressor and the amount of steady flow rate $(Q)$ was adjusted by a pressure regulator. The value of $Q$ was measured in collaboration with a Pitot tube and inclined leg alcohol-manometer. The airtight chamber was pressurized with a manual air pump and $p_{e}$ was measured by a 
pressure transmitter. The upstream pressure $\left(p_{1}\right)$ and downstream pressure $\left(p_{2}\right)$ of the tube were measured separately with pressure transducers. The oscillating flow velocity $\left(u_{o s}\right)$ measurement at the exit of airtight box was carried out through a hot-wire anemometer. The voltage outputs of pressure transducers and hot-wire anemometer were accumulated on a computer by means of a data acquisition system.

In the experiments Penrose tube which is made of latex and silicon rubber tube were used as collapsible tubes. The tube has been placed in the centre of the airtight box without giving any longitudinal tension. The coding for test tubes together with their inside diameter $\left(D_{0}\right)$, length $(L)$, tube-wall thickness $(h)$ and the ratio of inside radius $\left(R_{0}\right)$ to $h$ are given in table 1 . As an example for the coding system; $\mathrm{S}_{1, \mathrm{M}}$ defines silicone rubber tube for which $L=190.5 \mathrm{~mm}$ and $h=$ $1 \mathrm{~mm}$, namely subscript numbers indicate $h$ values while the subscript letters $\mathrm{L}$, $\mathrm{M}$ and $\mathrm{S}$ stand for long, medium and short tubes, respectively. The tubes of $R_{0} / h>10$ and $R_{0} / h<10$ are classified as thin-walled and thick-walled, respectively. Furthermore it has been shown in table 1 that $L / D_{0}$ ratio has three different values as 10, 7.5 and 5 which imply that a wide range depended on tube properties has been covered through the experimental study.

Table 1: The type and properties of collapsible tubes used in the experiments.

\begin{tabular}{|c|c|c|c|c|c|c|c|c|c|}
\hline Material & $\begin{array}{l}\text { Tube } \\
\text { Code }\end{array}$ & $L(\mathrm{~mm})$ & $\begin{array}{c}D_{0} \\
(\mathrm{~mm})\end{array}$ & $L / D_{0}$ & $h(\mathrm{~mm})$ & $R_{0} / h$ & Classification & $E(\mathrm{~Pa})$ & $K_{p}(\mathrm{~Pa})$ \\
\hline $\begin{array}{c}\text { Latex } \\
\text { (Penrose } \\
\text { Tube) } \\
\end{array}$ & $\begin{array}{l}P_{L} \\
P_{M} \\
P_{S} \\
\end{array}$ & $\begin{array}{c}254 \\
190.5 \\
127 \\
\end{array}$ & 25.4 & $\begin{array}{c}10 \\
7.5 \\
5\end{array}$ & 0.55 & 23.09 & thin-walled & $1.20 \mathrm{E}+06$ & 10.55 \\
\hline \multirow{2}{*}{$\begin{array}{l}\text { Silicone } \\
\text { Rubber }\end{array}$} & $\begin{array}{l}\mathrm{S}_{1, \mathrm{~L}} \\
\mathrm{~S}_{1, \mathrm{M}} \\
\mathrm{S}_{1, \mathrm{~S}}\end{array}$ & $\begin{array}{c}254 \\
190.5 \\
127\end{array}$ & 25.4 & $\begin{array}{c}10 \\
7.5 \\
5\end{array}$ & 1 & 12.7 & thin-walled & $2.03 \mathrm{E}+06$ & 107.31 \\
\hline & $\begin{array}{l}\mathrm{S}_{2, \mathrm{~L}} \\
\mathrm{~S}_{2, \mathrm{M}} \\
\mathrm{S}_{2, \mathrm{~S}}\end{array}$ & $\begin{array}{c}254 \\
190.5 \\
127\end{array}$ & 25.4 & $\begin{array}{c}10 \\
7.5 \\
5\end{array}$ & 2 & 6.35 & thick-walled & $2.03 \mathrm{E}+06$ & 858.48 \\
\hline
\end{tabular}

The modulus of elasticity of the tube material $(E)$ value, which was determined in the axial force-deflection test, is given in table 1. The parameter of $K_{p}$ seen in the last column of table 1 is the circumferential bending stiffness of the test tubes. It can be determined by the following relationship:

$$
K_{p}=\frac{1}{12} E \frac{\left(h / R_{0}\right)^{3}}{\left(1-v^{2}\right)}
$$


where $v$ is Poisson's ratio of the test tube material and it was assumed to be 0.48 which is an approximate value for rubber materials. Eqn. (1) shows that $K_{p}$ has the unit of pressure, i.e., Pascal.

\section{Experimental measurements and results}

All of the collapsible tubes specified in table 1 have been tested separately and experimental measurements were taken at various $Q$ values. The experimental protocol was such that any $Q$ has been supplied steadily to the system initially at $p_{e}=0 \mathrm{kPa}$ (gauge) and $p_{e}$ was gradually increased subsequently. The measurements have been commenced as soon as flow suddenly becomes oscillating following a collapse on the tube wall. The external pressure value which causes self-excited oscillations to occur has been called as the external pressure at collapse $\left(p_{e, c}\right)$ in this study. The time-dependent values of $p_{1}, p_{2}$ and $u_{o s}$ at this instant were saved to the computer as voltage values which were then converted into pressure and velocity units according to the calibration results of the measuring instruments. The root mean square (RMS) of $p_{1}, p_{2}$ and $u_{o s}$ were calculated from those oscillating flow measurements and the obtained RMS values have been represented as $\bar{p}_{1}, \bar{p}_{2}, \bar{u}_{o s}$, respectively. In addition the frequency of the oscillations $(f)$ has been determined by carrying out Fast Fourier Transform (FFT) to the unsteady pressure or velocity measurement.

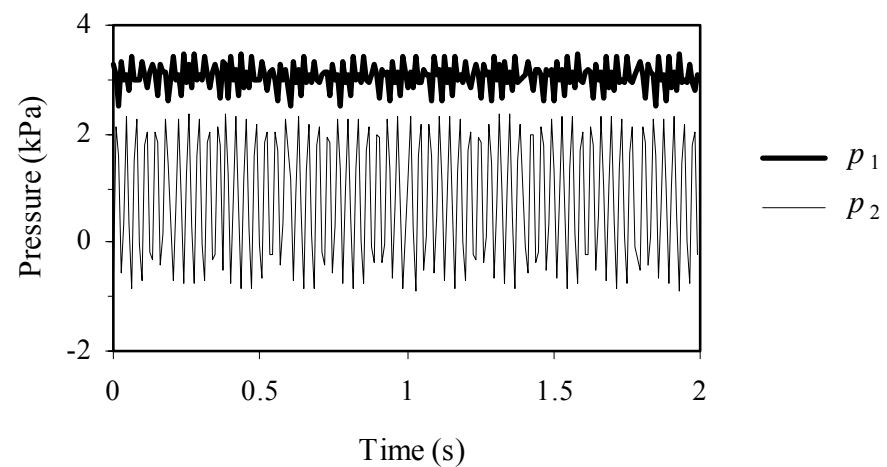

Figure 1: Variation of pressure with time at the onset of oscillations for the flow through $\mathrm{S}_{2, \mathrm{~S}}$ tube.

The variations of $p_{1}, p_{2}$ with time $(t)$ and $u_{o s}$ with $t$ are given as sample plots in fig. 1 and fig. 2 , respectively. These two figures had been obtained by testing $\mathrm{S}_{2, \mathrm{~S}}$ tube for an initial $Q$ value of $0.0147 \mathrm{~m}^{3} / \mathrm{s}$ and $p_{e, c}$ of $9.03 \mathrm{kPa}$ which triggers the oscillations at the supplied flow rate. Pressure and velocity change with time markedly as can be seen from these figures. Additionally it is clear in fig. 1 that $p_{2}$ value is more affected in comparison to $p_{1}$ value because $p_{1}$ changes as $2.75 \mathrm{kPa}<p_{1}<3.25 \mathrm{kPa}$, but $p_{2}$ fluctuates as $-1 \mathrm{kPa}<p_{2}<2 \mathrm{kPa}$. This can be 
evaluated as reasonable behaviour since pressure decreases in the flow direction, transmural pressure takes negative value near the exit of the tube and tube wall collapses at that point. The oscillations take place vigorously at that point near the tube exit and they even influence the tube inlet causing $p_{1}$ to change with time. Another result should be noticed is that $p_{2}$ sometimes takes negative values due to the oscillations. If variation of downstream velocity with time is examined in fig. 2 it can be remarked that $5 \mathrm{~m} / \mathrm{s}<u_{o s}<40 \mathrm{~m} / \mathrm{s}$. The experimental data in fig. 1 and fig. 2 had been evaluated and RMS values of the measurements were determined as $\bar{p}_{1}=3.01 \mathrm{kPa}, \bar{p}_{2}=1.38 \mathrm{kPa}$ and $\bar{u}_{o s}=27.84 \mathrm{~m} / \mathrm{s}$.

By applying FFT to the data of fig. 1, frequency of the oscillations was determined to be as $f=26.98 \mathrm{~Hz}$. It is noted that applying FFT to the variation $p_{1}, p_{2}$ or $u_{o s}$ does not change $f$ value as expected.

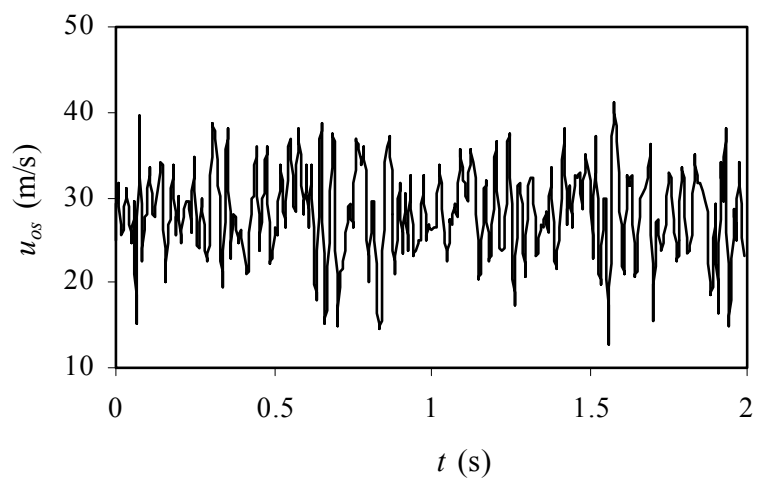

Figure 2: Variation of velocity with time at the onset of oscillations for the flow through $\mathrm{S}_{2, \mathrm{~s}}$ tube.

\subsection{Determination of the effect of $h$}

All of the collapsible tubes in table 1 have been tested by Oruç [10] similarly to the measurements (fig. 1 and fig. 2) cited above for $S_{2, S}$ tube. The overall effect of $h$ on $p_{e, c}$ is given as plots in fig. 3. It is easily said by examining fig. 3 that the thicker tube wall causes $p_{e, c}$ to increase at a known $Q$ value. For instance at $Q=0.0101 \mathrm{~m}^{3} / \mathrm{s}, p_{e, c}$ is approximately $0.25 \mathrm{kPa}, 2 \mathrm{kPa}$ and $9 \mathrm{kPa}$ for the flow through $\mathrm{P}_{\mathrm{L}}, \mathrm{S}_{1, \mathrm{~L}}$ and $\mathrm{S}_{2, \mathrm{~L}}$ tubes, respectively. This behaviour is also valid at other $Q$ values. It is again seen from fig. 3 that as $h$ becomes greater, $p_{e, c}$ also increases seriously. Furthermore, $p_{e, c}$ is nearly between $0.25 \mathrm{kPa}$ and $2 \mathrm{kPa}$ for all $Q$ values and all lengths of Penrose tubes in fig. 3. Similarly, it is changing between $2 \mathrm{kPa}$ and $3 \mathrm{kPa}$ for $\mathrm{S}_{1, \mathrm{~L}}, \mathrm{~S}_{1, \mathrm{M}}$ and $\mathrm{S}_{1, \mathrm{~S}}$ tubes. However $p_{e, c}$ range has increased seriously for $2 \mathrm{~mm}$ thick silicone rubber tubes as $8 \mathrm{kPa}<p_{e, c}<10 \mathrm{kPa}$, approximately regardless of $Q$. 


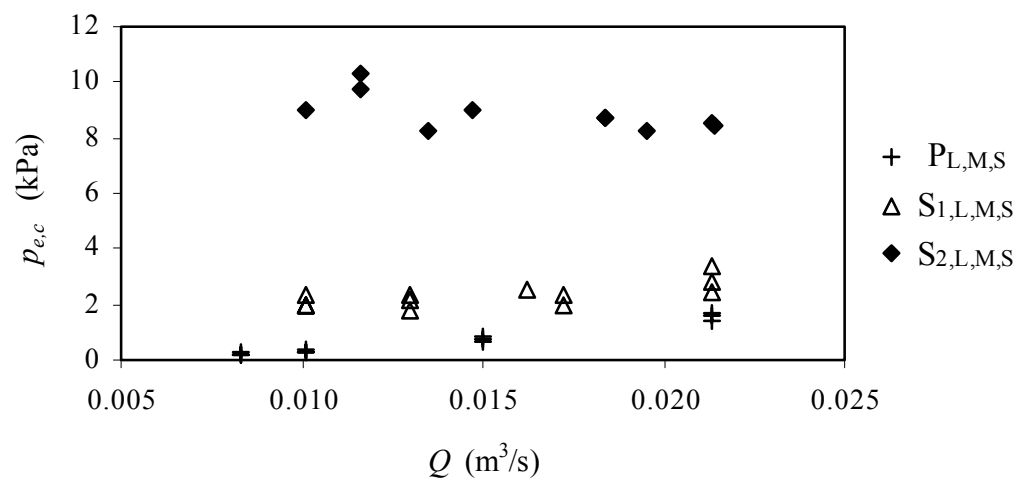

Figure 3: Variation of $p_{e, c}$ with $Q$ to show the effect of thickness of the test tubes at the onset of oscillations.

The effect of $h$ on $f$ is shown with the plots given in fig. 4. It seems that $f$ increases with increasing $h$ at a given $Q$ value. For example in fig. $4, f=20 \mathrm{~Hz}$ at $Q=0.0101 \mathrm{~m}^{3} / \mathrm{s}$ for $\mathrm{S}_{1, \mathrm{~L}}$ tube, however $f=30 \mathrm{~Hz}$ for $\mathrm{S}_{2, \mathrm{~L}}$ tube. It may be deduced by referring to fig. 4 that $f$ is in the range of $20 \mathrm{~Hz}$, approximately for all lengths of Penrose tubes. However a range of $20 \mathrm{~Hz}<f<25 \mathrm{~Hz}$ is seen for all lengths of thin-walled silicone rubber tube. Finally it is clear that $f$ takes values as $25 \mathrm{~Hz}<f<35 \mathrm{~Hz}$ for thick-walled silicone rubber tubes of different lengths. As a summary to explain the influence of $h$ it can be concluded that as $h$ increases $f$ and especially $p_{e, c}$ also increases.

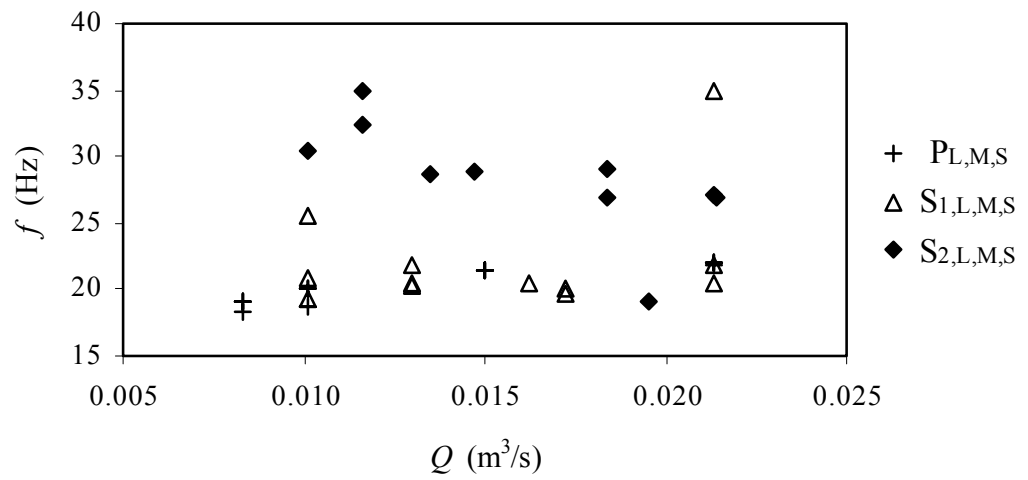

Figure 4: Variation of $f$ with $Q$ to show the effect of thickness of the test tubes at the onset of oscillations. 


\subsection{Determination of the effect of $L$}

The influence of $L$ can be analysed by referring to fig. 5. Variation of $p_{e, c}$ with $Q$ is plotted only for Penrose tubes having different lengths as shown in fig. 5. It can be said that $L$ has no much significance on $p_{e, c}$. Nevertheless, $p_{e, c}$ slightly increases as $L$ decreases. This can be verified at $Q=0.0213 \mathrm{~m}^{3} / \mathrm{s}$ case. Thus it can be concluded that $L$ has some effect on the determination of $p_{e, c}$. Higher $p_{e, c}$ necessary for a shorter tube means that as the tube becomes shorter it will be more difficult to collapse it, since the shorter tube may behave like a rigid tube.

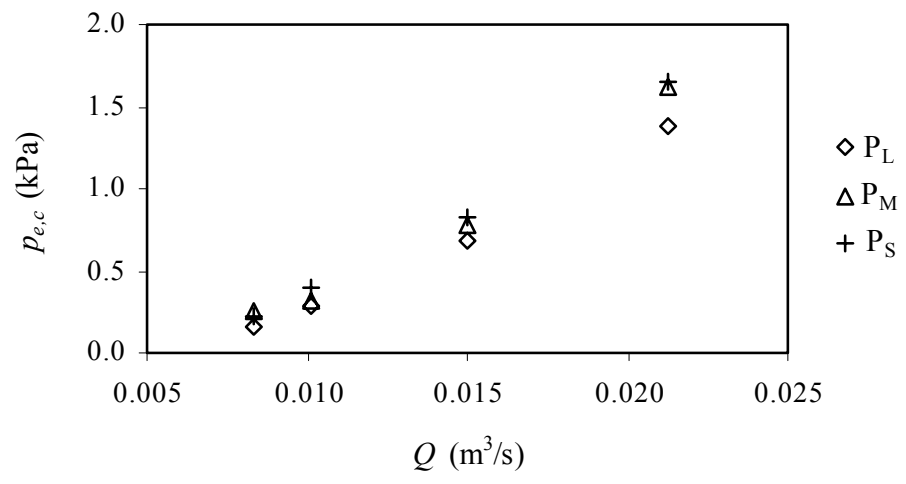

Figure 5: Variation of $p_{e, c}$ with $Q$ to show the effect of $L$ for Penrose tubes at the onset of oscillations.

\subsection{Evaluation of experimental results in terms of dimensionless parameters}

In this section the experimental measurement results were studied to express them as non-dimensional parameters. So that the dimensionless Womersley number $(W)$ can be expressed at the first step with the following equation:

$$
W=\sqrt{\left(2 \pi f R_{0}^{2} / v_{f}\right)}
$$

where $v_{f}=1.5 \times 10^{-5} \mathrm{~m}^{2} / \mathrm{s}$ is the kinematic viscosity of the fluid (air). Eqn. (2) has been plotted with respect to another dimensionless parameter as

$$
\left(\bar{p}_{2}-p_{e, c}\right) /\left(\bar{p}_{1}-\bar{p}_{2}\right)
$$

and the experimental results are shown in fig. 6 for all of the test tubes. This figure clearly indicates for the Penrose tubes that $W$ linearly increases as the nondimensional pressure term defined by eqn. (3) decreases. Furthermore the behaviour seems to be not so much affected by the length of Penrose tubes. For example $W$ is 35.5 and 39 corresponding to $\left(\bar{p}_{2}-p_{e, c}\right) /\left(\bar{p}_{1}-\bar{p}_{2}\right)$ values of 3.5 and 0.25 , respectively regardless of $L$. 


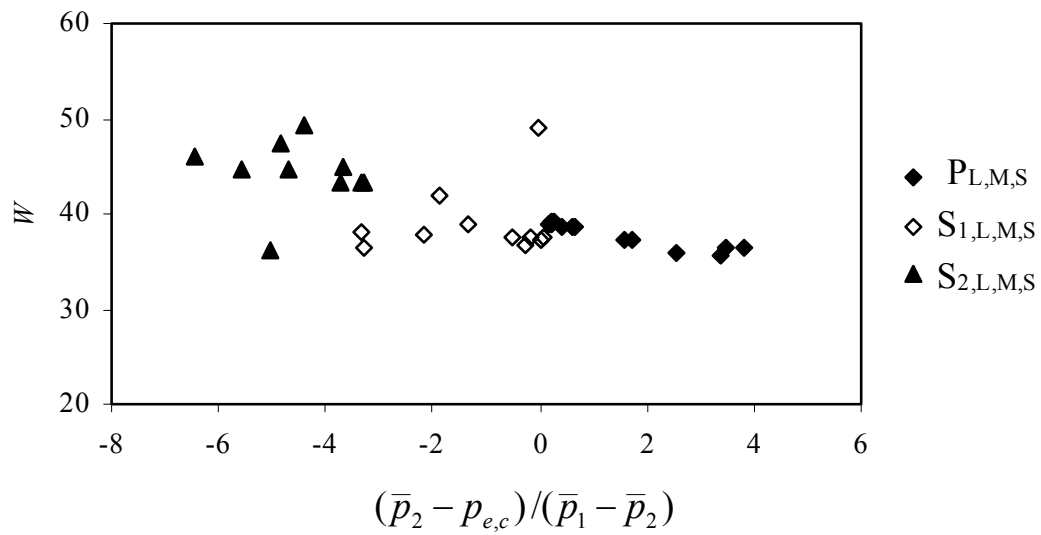

Figure 6: Variation of Womersley number with dimensionless downstream transmural pressure at the onset of oscillations.

If thin-walled silicone rubber tubes are examined in fig. 6 , it can be said that $W$ is not affected so much by the increase or decrease of $\left(\bar{p}_{2}-p_{e, c}\right) /\left(\bar{p}_{1}-\bar{p}_{2}\right)$ and it changes as $35<W<40$. However there is only one experimental data which has a $W$ of 50 related to $\mathrm{S}_{1, \mathrm{~L}}$ tube. This should be due to the higher $f$ value measured in the experiment. Similarly $W$ has a value between 45 and 50 related to the experiments with thick-walled silicone rubber tubes in fig. 6. It is clear that $W$ slightly increases as $h$ increases. It is also understood that experimental results with Penrose tubes figures out that the dimensionless pressure parameter defined by eqn. (3) is in the positive range. However it has always negative values for the results obtained from testing of silicone rubber tubes. In addition the effect of $L$ on the on the variation of $W$ with $\left(\bar{p}_{2}-p_{e, c}\right) /\left(\bar{p}_{1}-\bar{p}_{2}\right)$ seems to be insignificant.

The next dimensionless term which is related to mean oscillating velocity has been selected as

$$
\bar{u}_{o s} / \sqrt{K_{p} / \rho_{f}}
$$

where $\rho_{f}$ is density of the fluid and $K_{p}$ is computed by using eqn. (1). Note that $\sqrt{K_{p} / \rho_{f}}$ has the unit of velocity and eqn. (4) is therefore dimensionless.

In fig. 7, the dimensionless velocity has been plotted versus non-dimensional pressure term defined by eqn. (3). A cursory survey of this figure for the Penrose tubes points out that dimensionless mean oscillating velocity increases as the dimensionless mean downstream transmural pressure decreases regardless of $L$. There is a range of $5<\bar{u}_{o s} / \sqrt{K_{p} / \rho_{f}}<17.5$ corresponding to dimensionless mean downstream transmural pressure range between 4 and 2.5 related to the test with Penrose tubes. The result obtained from experiment with thin and thick- 
walled silicone rubber tubes is also shown in fig. 7. In contrary to the result obtained from the test with Penrose tube, dimensionless velocity increases with higher dimensionless transmural pressure. In this case the effect of $L$ is also not so much important. However it is noted that the thin-walled silicone rubber tube has a grater value of dimensionless velocity. In other words, the result with thick-walled silicone rubber tube gives $0.5<\bar{u}_{o s} / \sqrt{K_{p} / \rho_{f}}<1.5$, but it yields as $1.5<\bar{u}_{o s} / \sqrt{K_{p} / \rho_{f}}<4$ for the experiment with thin-walled silicone rubber tubes. It should be emphasized that the dimensionless velocity values are higher for Penrose tubes in comparison with silicone rubber tubes.

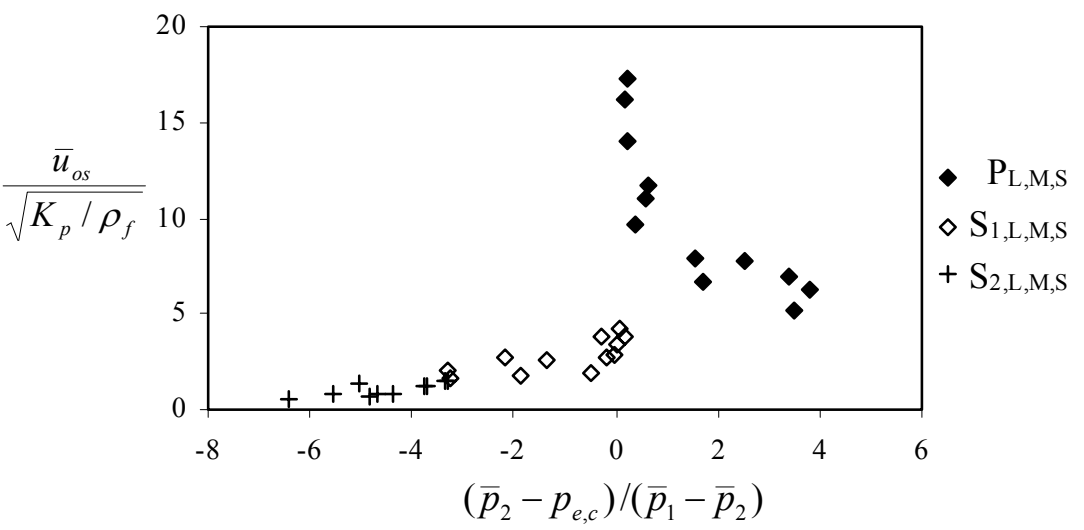

Figure 7: Mean oscillating flow velocity versus downstream transmural pressure in dimensionless form at the onset of oscillations.

\section{Conclusion}

In this paper, results of the experimental measurements related to airflow through collapsible tubes are presented in detail. The measurements correspond to the instant at which self-excited oscillations arise due to the collapse of the tube under the applied external pressure. It has been found that pressure and velocity signals fluctuate seriously with time during oscillations. Also $p_{2}$ seems to be more affected by the oscillations in comparison with $p_{1}$ (fig. 1). Furthermore frequency of the oscillations may be determined by FFT analysis of velocity or pressure data. The main experimental results are illustrated by the plots from fig. 3 to fig. 5 . It has been realized by examining these figures that $p_{e, c}$ and $f$ are influenced by the tube properties such as $h, L, E$ as well as flow rate. It is seen that $f$ and particularly $p_{e, c}$ are much more affected by $h$ in comparison with $L$. Therefore all of these parameters suggested to be carefully taken into consideration in collapsible tube flow investigations.

Moreover the relationships between some dimensionless parameters are presented as shown in fig. 6 and fig. 7. It is seen that these dimensionless 
parameters have also been affected by $Q, L, h$ as well as the tube's material type. The expressed dimensionless parameters are significant because of the fact that they include many of the factors related to flow $\left(\bar{p}_{1}, \bar{p}_{2}, f, p_{e, c}, \bar{u}_{o s}, \rho_{f}, v_{f}\right)$ and tube $\left(D_{0}, L, h, K_{p}\right)$. Therefore these non-dimensional terms should be useful in the similar investigations.

\section{Acknowledgements}

The presented study is being financially supported by TUBITAK with the research project no.105M301. The first author would like to express his special thanks to Mr. Osman Oruç and Dr. Orhan Çakır due to their contributions to this study.

\section{References}

[1] Cancelli, C. \& Pedley, T.J., A separated flow model for collapsible tube oscillations. Journal of Fluid Mechanics, 157, pp. 375-404, 1985.

[2] Conrad, W.A., Pressure-flow relationships in collapsible tubes. IEEE Transactions on Biomedical Engineering, BME-16, pp. 284-295, 1969.

[3] Ohba, K., Sakurai, A. \& Oka, J., Laser doppler measurement of local flow field in collapsible tube during self-excited oscillation. JSME International Journal, Series C, 40, pp. 665-670, 1997.

[4] Gavriely, N., Shee, T.R., Cugell, D.W. \& Grotberg, J. B., Flutter in flow limited collapsible tubes: a mechanism for generation of wheezes. Journal of Applied Physiology, 66, pp. 2251-2261, 1989.

[5] Bertram, C.D., Raymond, C.J. \& Butcher, K.S.A., Oscillations in a collapsed-tube analog of the brachial artery under a sphygmomanometer cuff. ASME Journal of Biomechanical Engineering, 111, pp. 185-191, 1989.

[6] Griffiths, D.J., Hydrodynamics of male micturition. Part I: theory of steady flow through elastic-walled tubes. Medical and Biological Engineering and Computing, 9, pp. 581-588, 1971.

[7] Gavriely, N., Palti, Y., Alroy, G. \& Grotberg, J.B., Measurement and theory of wheezing breath sounds. Journal of Applied Physiology, 57, pp. 481-492. 1984.

[8] Gavriely, N. \& Jensen, O.E., Theory and measurements of snores. Journal of Applied Physiology, 74, pp. 2828-2837, 1993.

[9] Oruç, V. \& Çarpınlığlu, M.Ö., A test rig for the investigation of airflow through collapsible tubes. Proceedings of the Institution of Mechanical Engineers, Part C: Journal of Mechanical Engineering Science (in press).

[10] Oruç, V., An experimental investigation on the analysis of flow dynamics in collapsible elastic tubes. Ph.D. thesis, University of Gaziantep, Turkey, 2007. 